\title{
Bericht zur Lage der Bibliotheken 2020 erschienen
}

\author{
„Die positive Entwicklung von Bibliotheken als \\ Innovationstreiber darf nicht durch Sparmaßnahmen \\ in Folge der Pandemie gefährdet werden.“
}

https://doi.org/10.1515/bd-2021-0003

Die Corona-Pandemie bestimmt in diesem Jahr maßgeblich die Arbeit von Bibliotheken. Dank ihrer zahlreichen Online-Services konnten Bibliotheken die Bürger*innen auch während des pandemiebedingten Lockdowns weiter mit Medien, Bildungsangeboten und Informationen versorgen. In dieser Situation wurden rasch zusätzlich viele neue digitale - kontaktarme - Bibliotheksangebote geschaffen. Dass Bibliotheken mit als erste Einrichtungen wieder öffnen durften, unterstreicht ihre Bedeutung für die Bürger`innen in Kommunen und Städten.

Der diesjährige „Bericht zur Lage der Bibliotheken“ befasst sich schwerpunktmäßig mit den Folgen der Corona-Pandemie. Derzeit ist nicht abzusehen, wie sich die finanzielle Situation der Kommunen und damit der Stadt- und Gemeindebibliotheken kurz- und mittelfristig entwickeln wird. Daher ist ein Fazit des Berichts, dass die Bewältigung der finanziellen Belastungen der Corona-Pandemie die Teilhabe der Bürger`innen an Bildung und Kultur sowie die Vermittlung von Informations- und Digitalkompetenz zu beinträchtigen droht, wenn die Bibliotheken nicht ausreichend finanziert und massive Einsparungen zu erwarten sind.

Weitere Themen des Berichts zur Lage sind u.a. der digitale Wandel in Bibliotheken, der Beitrag von Bibliotheken zur Erreichung der UN-Nachhaltigkeitsziele, der wachsende Bedarf an Infrastrukturen für Forschungsdaten sowie die Stärkung von Bibliotheken als Einrichtungen der Medienbildung.

Dazu Prof. Dr. Andreas Degkwitz, Bundesvorsitzender des Deutschen Bibliotheksverbandes e.V. (dbv): „Mit der Erfüllung ihres Bildungs- und Kulturauftrags leisten Bibliotheken einen elementaren Beitrag zum demokratischen Zusammenhalt in unserer Gesellschaft. Dies gilt in besonderer Weise für Krisen, wie wir sie gegenwärtig mit Corona erleben. Denn Bibliotheken tragen dazu bei, die Herausforderungen der Covid-19-Pandemie zu überwinden, indem sie Menschen dabei unterstützen, sich weiterzubilden, auszutauschen und zu informieren. Die positive Entwicklung von Bibliotheken als attraktive ,Dritte Orte und kompetente Innovationstreiber darf nicht durch Sparmaßnahmen als Folge der Pandemie 
zunichte gemacht werden. Gerade jetzt brauchen wir leistungsstarke Bibliotheken - für Bildungsgerechtigkeit und Teilhabechancen aller.“

Der „Bericht zur Lage der Bibliotheken 2020/2021“ kann hier heruntergeladen werden: https://www.bibliotheksverband.de/fileadmin/user_upload/DBV/ publikationen/Bericht_zur_Lage_2020_2021_WEB.pdf.

\section{Zum Hintergrund}

Einmal im Jahr veröffentlicht der Deutsche Bibliotheksverband e.V. (dbv) den „Bericht zur Lage der Bibliotheken“. Darin legt er Zahlen und Fakten zu Entwicklungen und zur Arbeit der Bibliotheken vor und formuliert bibliothekspolitische Forderungen.

\section{Der Deutsche Bibliotheksverband}

Der Deutsche Bibliotheksverband e.V. (dbv) vertritt mit seinen mehr als 2.100 Mitgliedern bundesweit rund 9.000 Bibliotheken mit 25.000 Beschäftigten und 11 Mio. Nutzer^innen. Sein zentrales Anliegen ist es, Bibliotheken zu stärken, damit sie allen Bürger`innen freien Zugang zu Informationen ermöglichen. Der Verband setzt sich ein für die Entwicklung innovativer Bibliotheksleistungen für Wissenschaft und Gesellschaft. Als politische Interessensvertretung unterstützt der dbv die Bibliotheken insbesondere auf den Feldern Informationskompetenz und Medienbildung, Leseförderung und bei der Ermöglichung kultureller und gesellschaftlicher Teilhabe für alle Bürger`innen.

Mehr Informationen finden Sie unter www.bibliotheksverband.de.

\section{Pressekontakt:}

Kristin Bäßler

Leitung Kommunikation / Pressesprecherin

Deutscher Bibliotheksverband e.V.

Fritschestr. 27-28 | 10585 Berlin

Tel.: 030/644 98 99-25 | E-Mail: baessler@bibliotheksverband.de

URL: www.bibliotheksverband.de 